\title{
Light charged Higgs boson production at the Large Hadron electron Collider
}

\author{
O. Flores-Sánchez \\ Departamento de Sistemas y Computación, Tecnológico Nacional de México, \\ Instituto Tecnológico de Puebla, Avenida Tecnológico num. 420, \\ Maravillas, C.P. 72220, Puebla, Puebla, Mexico \\ J. Hernández-Sánchez ${ }^{\dagger}$ and C. G. Honorato \\ Fac. de Cs. de la Electrónica, Benemérita Universidad Autónoma de Puebla, \\ Apartado Postal 1152, 72570 Puebla, Puebla, Mexico \\ S. Moretti ${ }^{\S}$ \\ School of Physics and Astronomy, University of Southampton, \\ Highfield, Southampton SO17 1BJ, United Kingdom, \\ and Particle Physics Department, Rutherford Appleton Laboratory, \\ Chilton, Didcot, Oxon OX11 OQX, United Kingdom \\ S. Rosado-Navarro \\ Fac. de Cs. Físico-Matemáticas, Benemérita Universidad Autónoma de Puebla, \\ Apartado Postal 1364, C.P. 72570 Puebla, Puebla, Mexico
}

(Received 20 November 2018; published 13 May 2019)

\begin{abstract}
We study the production of a light charged Higgs boson at the future Large Hadron electron Collider, through the process $e^{-} p \rightarrow \nu_{e} H^{-} q$, considering both decay channels $H^{-} \rightarrow b \bar{c}$ and $H^{-} \rightarrow \tau \bar{\nu}_{\tau}$ in the final state. We analyze these processes in the context of the two-Higgs doublet model type III and assess the Large Hadron electron Collider sensitivity to such $H^{-}$signals against a variety of both reducible and irreducible backgrounds. We confirm that prospects for $\mathrm{H}^{-}$detection in the two-Higgs doublet model type III are excellent, assuming standard collider energy and luminosity conditions.
\end{abstract}

DOI: 10.1103/PhysRevD.99.095009

\section{INTRODUCTION}

Now that a neutral Higgs boson has been discovered at the large hadron collider (LHC) by the ATLAS [1] and CMS [2] experiments, the standard model (SM) appears to be fully established. However, the SM-like limit of electroweak symmetry breaking (EWSB) dynamics induced by a Higgs potential exists in several beyond the SM (BSM) extensions of the Higgs sector. Notably, the two-Higgs doublet model (2HDM) in its versions type I, type II, type III (or Y), and type IV (or X), wherein flavor-changing

*omar.flores@itpuebla.edu.mx

jaime.hernandez@correo.buap.mx

*carlosg.honorato@correo.buap.mx

ss.moretti@soton.ac.uk

" sebastian.rosado@gmail.com

Published by the American Physical Society under the terms of the Creative Commons Attribution 4.0 International license. Further distribution of this work must maintain attribution to the author(s) and the published article's title, journal citation, and DOI. Funded by SCOAP. neutral currents (FCNCs) mediated by (pseudo)scalars can be eliminated under discrete symmetries [3], is an intriguing BSM candidate, owing to the fact that it implements the same fundamental doublet construct of the SM (albeit twice), assumes the same SM gauge group, and predicts a variety of new Higgs boson states that may be accessible at the LHC. In fact, another, very interesting kind of 2HDM is the one in which FCNCs can be controlled by a particular texture in the Yukawa matrices [4]. In particular, in previous papers, we have implemented a four-zero texture in a scenario which we have called 2HDM type III (2HDM-III) [5]. This model has a phenomenology which is very rich, which we studied at colliders in various instances [6-12], and some very interesting aspects, like flavor-violating quarks decays, which can be enhanced for neutral Higgs bosons with intermediate mass (i.e., below the top quark mass). In particular, we have studied the signal $\phi_{i}^{0} \rightarrow s \bar{b}+$ H.c. $\left(\phi_{i}^{0}=h, H\right)$ at the future $e p$ machine, the Large Hadron electron Collider (LHeC) $[7,8]$. Specifically, taking into account the characteristics of such a machine, we have established the leading charged 


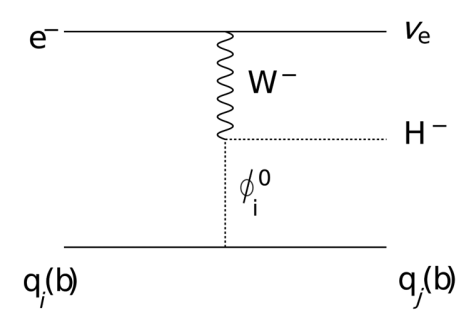

(a)

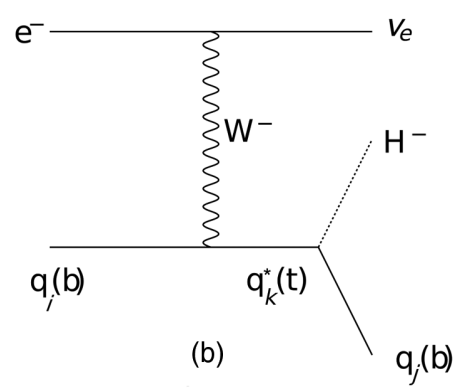

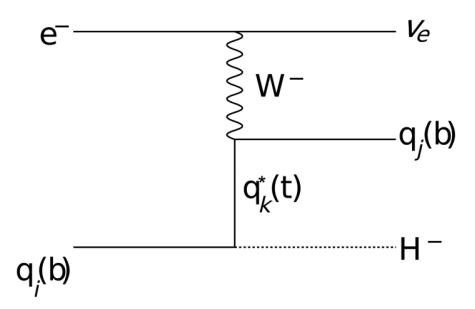

(c)

FIG. 1. Feynman diagrams for the $e^{-} p \rightarrow \nu_{e} H^{-} q$ process. Here, $\phi_{i}^{0}=h, H, A$, i.e., any of the neutral Higgs bosons of the BSM scenario considered here (see below).

current production process $e^{-} p \rightarrow \nu_{e} \phi_{i}^{0} q$ followed by the signature $\phi_{i}^{0} \rightarrow s \bar{b}+$ H.c., by considering $3 j+E_{T}$ as final state, where $j$ represents a jet and $E_{T}$ refers to missing transverse energy. Furthermore, in this model, the parameter space can avoid the current experimental constraints from flavor and Higgs physics, and a light charged Higgs boson is allowed [11], so that the decay $H^{-} \rightarrow b \bar{c}$ is enhanced and its branching ratio (BR) can be dominant. In fact, this channel has been also studied in a variety of multi-Higgs doublet models (MHDMs) [13,14], wherein the $\operatorname{BR}\left(H^{-} \rightarrow b \bar{c}\right) \approx 0.7-0.8$ and could afford one with a considerable gain in sensitivity to the presence of a $H^{-}$by tagging the $b$ quark.

Previously, we did a parton-level study of the process $e^{-} p \rightarrow \nu_{e} H^{-} b$ followed by the signal $H^{-} \rightarrow b \bar{c}$ [6]. The paper presented several benchmark model scenarios but they were not subject to the most recent experimental results from Higgs boson physics, in particular, the latest measurement of the signal strength of $h \rightarrow b \bar{b}$ [15]. The complete analysis and the reconstruction of this signal at the detector level for the $\mathrm{LHeC}$ machine is presented here. Furthermore, in this work, we tension the $H^{-} \rightarrow b \bar{c}$ channel against the $H^{-} \rightarrow \tau \bar{\nu}_{\tau}$ one and contrast the scope of the two modes in order to establish the sensitivity of the LHeC [16,17] to the presence of light charged Higgs bosons of the 2HDM-III. Specifically, we study the process $e^{-} p \rightarrow \nu_{e} H^{-} q$ (Fig. 1), in which $q$ represents both a light flavor $q_{l}=d, u, s, c$ and a $b$ quark, followed by the decays $H^{-} \rightarrow b \bar{c}$ and $\tau \bar{\nu}_{\tau}$ (assuming in turn a leptonic decay of the $\tau$ into an electron or muon). In the former case, we compare the signal yield against that of the main backgrounds: $\nu 3 j$, $\nu 2 b j, \nu 2 j b$, and $\nu t b$. In the latter case, we consider instead the backgrounds $\nu j \ell \nu$ and $\nu b \ell \nu$. (All relevant backgrounds are schematically represented in Figs. 2 and 3.)

The plan of this paper is as follows. In the next section, we describe the 2HDM-III. Then, we discuss our results. Finally, we conclude.

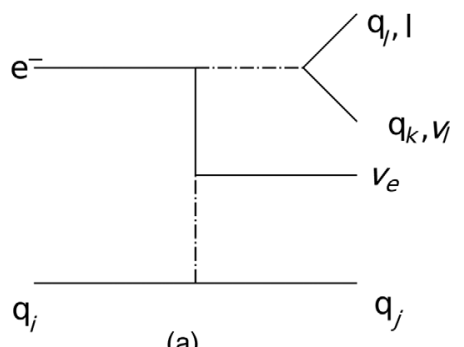

(a)

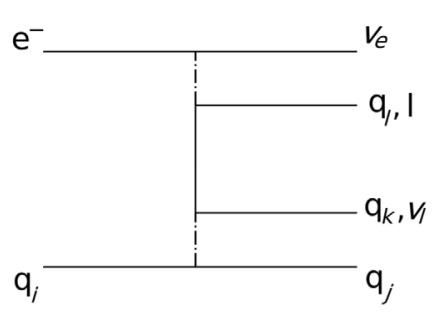

(d)
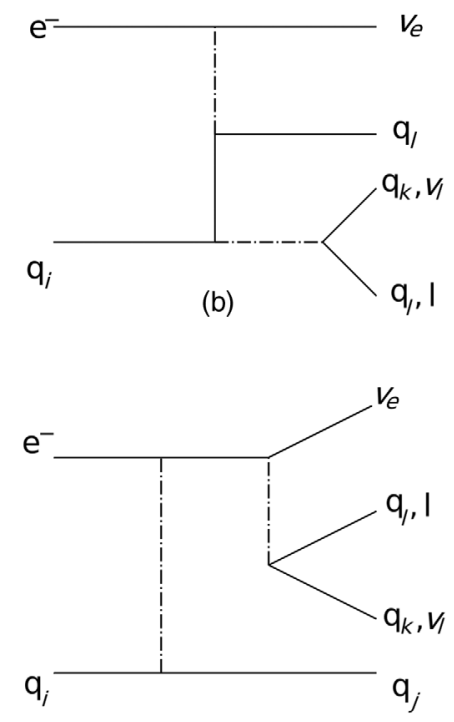

(e)

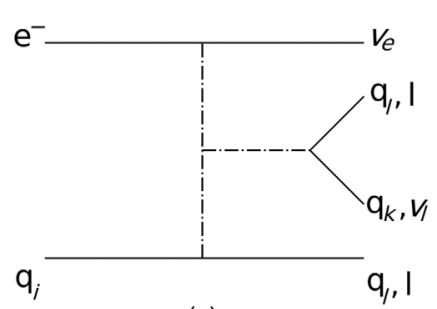

(c)

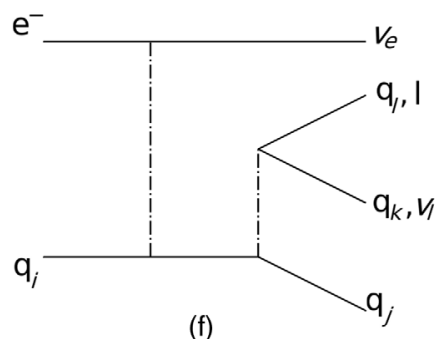

(f)

FIG. 2. Feynman diagrams for the $\nu_{e} j j j, \nu_{e} b j j$, and $\nu_{e} b b j$ backgrounds (the change $q_{l} \leftrightarrow l$ and $q_{k} \leftrightarrow \nu_{l}$ represents the $\nu_{e} \nu_{l} l j$ and $\nu_{e} \nu_{l} l b$ backgrounds). Dashed-dotted lines represent boson fields: (pseudo)scalars and EW gauge bosons. 


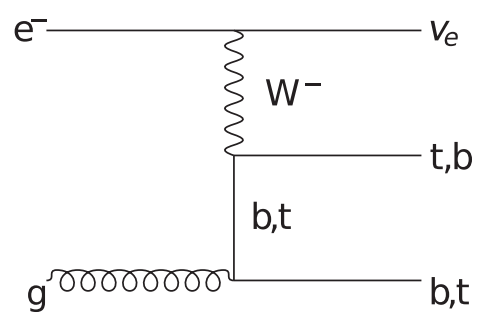

FIG. 3. Feynman diagrams for the $\nu_{e} b t$ background.

\section{2HDM-III}

In the 2HDM-III, the two Higgs (pseudo)scalar doublets, $\Phi_{1}^{\dagger}=\left(\phi_{1}^{-}, \phi_{1}^{0 *}\right)$ and $\Phi_{2}^{\dagger}=\left(\phi_{2}^{-}, \phi_{2}^{0 *}\right)$, have hypercharge +1 , and both couple to all fermions. Here, a specific four-zero texture is implemented as an effective flavor symmetry in the Yukawa sector, which we have shown previously to be the mechanism controlling FCNCs. Therefore, it is not necessary to consider discrete symmetries in the Higgs potential $[10,11]$. Then, one can study the most general $S U(2)_{L} \times U(1)_{Y}$ invariant (pseudo)scalar potential given by

$$
\begin{aligned}
V\left(\Phi_{1}, \Phi_{2}\right)= & \mu_{1}^{2}\left(\Phi_{1}^{\dagger} \Phi_{1}\right)+\mu_{2}^{2}\left(\Phi_{2}^{\dagger} \Phi_{2}\right)-\left(\mu_{12}^{2}\left(\Phi_{1}^{\dagger} \Phi_{2}+\text { H.c. }\right)\right) \\
& +\frac{1}{2} \lambda_{1}\left(\Phi_{1}^{\dagger} \Phi_{1}\right)^{2}+\frac{1}{2} \lambda_{2}\left(\Phi_{2}^{\dagger} \Phi_{2}\right)^{2} \\
& +\lambda_{3}\left(\Phi_{1}^{\dagger} \Phi_{1}\right)\left(\Phi_{2}^{\dagger} \Phi_{2}\right)+\lambda_{4}\left(\Phi_{1}^{\dagger} \Phi_{2}\right)\left(\Phi_{2}^{\dagger} \Phi_{1}\right) \\
& +\left(\frac{1}{2} \lambda_{5}\left(\Phi_{1}^{\dagger} \Phi_{2}\right)^{2}+\lambda_{6}\left(\Phi_{1}^{\dagger} \Phi_{1}\right)\left(\Phi_{1}^{\dagger} \Phi_{2}\right)\right. \\
& \left.+\lambda_{7}\left(\Phi_{2}^{\dagger} \Phi_{2}\right)\left(\Phi_{1}^{\dagger} \Phi_{2}\right)+\text { H.c. }\right),
\end{aligned}
$$

where we assume all parameters to be real, ${ }^{1}$ including the vacuum expectation values (VEVs) of the Higgs fields; hence, there is no $C P$-violating dynamics. Usually, when a discrete symmetry $\Phi_{1} \rightarrow \Phi_{1}$ and $\Phi_{2} \rightarrow-\Phi_{2}$ is considered, the $\lambda_{6}$ and $\lambda_{7}$ parameters are absent. In general, for two (complex) doublet fields, there are eight fields, but only five of them are physical (pseudo)scalar ("Higgs") fields, which correspond to two neutral $C P$-even bosons, $h$ (the light one) and $H$ (the heavy one); one neutral $C P$-odd boson $A$; and two charged bosons $H^{ \pm}$. The mixing angle $\alpha$ of the two neutral $C P$-even bosons $h$ and $H$ is another parameter of the 2HDM. In total, the 2HDM can be described by the parameters $\alpha$ and $\beta$ (where $\tan \beta$ is the ratio of the VEVs of the two doublet fields) and the masses of the five Higgs particles. With these inputs, one can estimate all the parameters that are present in the scalar potential.

The other hand, when mass matrices with a four-zero texture are considered instead, one can keep the terms

\footnotetext{
${ }^{1}$ The $\mu_{12}^{2}, \lambda_{5}, \lambda_{6}$, and $\lambda_{7}$ parameters could be complex in general.
}

proportional to $\lambda_{6}$ and $\lambda_{7}$. Besides, we have shown that these parameters play a relevant role in one-loop processes because self-interactions of Higgs bosons are sizeable. In contrast, the electroweak (EW) parameter $\rho=m_{W}^{2} / m_{Z}^{2} \cos _{W}^{2}$ receives large one-loop corrections directly by the mass difference between charged Higgs and $C P$-even/-odd masses, which can be large, irrespective of the value of $\lambda_{6}$ and $\lambda_{7}$ [9]. Specifically, the underlying custodial symmetry (twisted custodial symmetry) is broken when the difference of the scalars masses $m_{H^{ \pm}}-m_{A}\left(m_{H^{ \pm}}-m_{H}\right)$ is substantial. Yet a surviving model is possible when the parameter $\rho \approx 1$ [18-20], so we have enforced this condition. In such a case, in agreement with Ref. [18], when taking the SM-like scenario [when $\cos (\beta-\alpha) \rightarrow 0$ ], we can obtain $m_{A}^{2}-m_{H}^{2}=O\left(v^{2}\right)$, and under these assumptions, a scenario with a light mass for the charged Higgs boson is feasible. Furthermore, the mass splitting among $H^{ \pm}, H$, and $A$ must also be reconciled with the general expressions of the oblique parameters $S, T$, and $U$ when the Higgs potential embeds $C P$ conservation [21] (the socalled EW precisions observables [22]). These EW bounds are implemented in the benchmarks scenarios chosen and discussed in the next section.

In our construction, the Yukawa Lagrangian is given by [11]

$$
\begin{aligned}
\mathcal{L}_{Y}= & -\left(Y_{1}^{u} \bar{Q}_{L} \tilde{\Phi}_{1} u_{R}+Y_{2}^{u} \bar{Q}_{L} \tilde{\Phi}_{2} u_{R}+Y_{1}^{d} \bar{Q}_{L} \Phi_{1} d_{R}\right. \\
& \left.+Y_{2}^{d} \bar{Q}_{L} \Phi_{2} d_{R}+Y_{1}^{l} \bar{L}_{L} \tilde{\Phi}_{1} l_{R}+Y_{2}^{l} \bar{L}_{L} \tilde{\Phi}_{2} l_{R}\right),
\end{aligned}
$$

where $\tilde{\Phi}_{1,2}=i \sigma_{2} \Phi_{1,2}^{*}$. So, the fermion mass matrices after EWSB are given by $M_{f}=\frac{1}{\sqrt{2}}\left(v_{1} Y_{1}^{f}+v_{2} Y_{2}^{f}\right), f=u, d, l$, where we have assumed that both Yukawa matrices $Y_{1}^{f}$ and $Y_{2}^{f}$ have the aforementioned four-zero-texture form and are Hermitian. After diagonalization, $\bar{M}_{f}=V_{f L}^{\dagger} M_{f} V_{f R}$, one has $\bar{M}_{f}=\frac{1}{\sqrt{2}}\left(v_{1} \tilde{Y}_{1}^{f}+v_{2} \tilde{Y}_{2}^{f}\right)$, where $\tilde{Y}_{i}^{f}=V_{f L}^{\dagger} Y_{i}^{f} V_{f R}$. One can obtain a good approximation for the product $V_{q} Y_{n}^{q} V_{q}^{\dagger}$ by expressing the rotated matrix $\tilde{Y}_{n}^{q}$ as [11],

$$
\left[\tilde{Y}_{n}^{q}\right]_{i j}=\frac{\sqrt{m_{i}^{q} m_{j}^{q}}}{v}\left[\tilde{\chi}_{n}^{q}\right]_{i j}=\frac{\sqrt{m_{i}^{q} m_{j}^{q}}}{v}\left[\chi_{n}^{q}\right]_{i j} e^{i \vartheta_{i j}^{q}},
$$

where the $\chi \mathrm{s}$ are unknown dimensionless parameters of the model. Following the procedure of Ref. [11], we can get a generic expression for the couplings of the charged Higgs bosons to the fermions as

$$
\begin{aligned}
\mathcal{L}^{\bar{f}_{i} f_{j} \phi}= & -\left\{\frac{\sqrt{2}}{v} \bar{u}_{i}\left(m_{d_{j}} X_{i j} P_{R}+m_{u_{i}} Y_{i j} P_{L}\right) d_{j} H^{+}\right. \\
& \left.+\frac{\sqrt{2} m_{l_{j}}}{v} Z_{i j} \bar{\nu}_{L} l_{R} H^{+}+\text {H.c. }\right\},
\end{aligned}
$$


TABLE I. The parameters $X, Y$, and $Z$ of the 2HDM-III defined in the Yukawa interactions when $\chi_{i j}^{f}=0$ so as to recover the standard four types of 2HDM.

\begin{tabular}{lrlr}
\hline \hline 2HDM-III & $X$ & $Y$ & $Z$ \\
\hline 2HDM type I & $-\cot \beta$ & $\cot \beta$ & $-\cot \beta$ \\
2HDM type II & $\tan \beta$ & $\cot \beta$ & $\tan \beta$ \\
2HDM type X & $-\cot \beta$ & $\cot \beta$ & $\tan \beta$ \\
2HDM type Y & $\tan \beta$ & $\cot \beta$ & $-\cot \beta$ \\
\hline \hline
\end{tabular}

where $X_{i j}, Y_{i j}$, and $Z_{i j}$ are defined as

$$
\begin{aligned}
X_{i j} & =\sum_{l=1}^{3}\left(V_{\mathrm{CKM}}\right)_{i l}\left[X \frac{m_{d_{l}}}{m_{d_{j}}} \delta_{l j}-\frac{f(X)}{\sqrt{2}} \sqrt{\frac{m_{d_{l}}}{m_{d_{j}}}} \tilde{\chi}_{l j}^{d}\right], \\
Y_{i j} & =\sum_{l=1}^{3}\left[Y \delta_{i l}-\frac{f(Y)}{\sqrt{2}} \sqrt{\frac{m_{u_{l}}}{m_{u_{i}}}} \tilde{\chi}_{i l}^{u}\right]\left(V_{\mathrm{CKM}}\right)_{l j}, \\
Z_{i j}^{l} & =\left[Z \frac{m_{l_{i}}}{m_{l_{j}}} \delta_{i j}-\frac{f(Z)}{\sqrt{2}} \sqrt{\frac{m_{l_{i}}}{m_{l_{j}}}} \tilde{\chi}_{i j}^{l}\right],
\end{aligned}
$$

where $f(a)=\sqrt{1+a^{2}}$ and the parameters $X, Y$, and $Z$ are arbitrary complex numbers, which can be related to $\tan \beta$ or $\cot \beta$ when $\chi_{i j}^{f}=0$ [11], thus recovering the standard four types of the 2 HDM (see Table I), ${ }^{2}$ and the Higgs-fermionfermion $(\phi f f)$ couplings in the 2HDM-III are written as $g_{2 \mathrm{HDM}-\mathrm{III}}^{\phi f f}=g_{2 \mathrm{HDM}-\mathrm{any}}^{\phi f f}+\Delta g$, where $g_{2 \mathrm{HDM}-\text { any }}^{\phi f f}$ is the coupling $\phi f f$ in any of the 2HDMs with discrete symmetry and $\Delta g$ is the contribution of the four-zero texture. Finally, we have also pointed out that this Lagrangian can represent a MHDM or an aligned 2HDM with additional flavor physics in the Yukawa matrices $[10,11]$.

\section{BENCHMARK SCENARIOS}

The 2HDM-III has been constrained previously by us, see Refs. [6-12], by taking into account both flavor and Higgs physics as well as EW precision observables (e.g., the oblique parameters) plus theoretical bounds such as vacuum stability, unitarity, as well as perturbativity. In particular, for our present study, we confine ourselves to the parameter space region where $m_{h}=125 \mathrm{GeV}$ (hence, $h$ is the SM-like Higgs boson), with $m_{A}=100 \mathrm{GeV}$, $180 \mathrm{GeV}<m_{H}<260 \mathrm{GeV}$, and $100 \mathrm{GeV}<m_{H^{ \pm}}<170 \mathrm{GeV}$, further assuming $\cos (\beta-\alpha)=0.1,0.5$. We have fixed the oblique parameter $U=0$, since that $U$ is suppressed by a factor of order the new physics scale $\Lambda^{2}$ compared to the parameters $S$ and $T$ [22], where $S=0.02 \pm 0.07$ and $T=0.06 \pm 0.06$. In general, the parameter space of the

\footnotetext{
${ }^{2}$ So, we will refer to these 2HDM-III "incarnations" as 2HDMIII like- $\chi$ scenarios, where $\chi=\mathrm{I}, \mathrm{II}, \mathrm{X}$, and $\mathrm{Y}$ (to be defined below).
}

2HDM-III with the four-zero texture considered here is fully compatible with the SM-like Higgs boson discovery [23], as we have implemented the same analysis of Refs. [9-11], wherein the radiative decays $h \rightarrow \gamma \gamma, \gamma Z$ were studied and the impact of the charged Higgs bosons flowing in the corresponding loops was analyzed in detail. We adapt that study here by taking into account the most recent experimental data from the LHC for these two decay modes [24-28]: upon applying these filters, the mass of the light charged Higgs boson is constrained in the range $110 \mathrm{GeV}<m_{H^{ \pm}}<170 \mathrm{GeV}$ with $\cos (\beta-\alpha)=0.1,0.5$. However, fermiophobic couplings for a charged Higgs boson with mass in the range $79 \mathrm{GeV}<m_{H^{ \pm}}<100 \mathrm{GeV}$ are allowed in light of the given experimental constraints. Furthermore, recent experimental bounds from flavor physics are considered here, following the analysis of Refs. [10,11,29], in which the parameter space of the model is bound by leptonic and semileptonic meson decays, being the inclusive decay $B \rightarrow X_{s} \gamma, B_{0}-B_{0}$, and $K_{0}-K_{0}$ mixing as well as $B_{s} \rightarrow \mu^{+} \mu^{-}$transitions the strongest constraints available. One can further get a scenario in which a rather light charged Higgs mass is feasible because the Yukawa-texture effects enter directly in the amplitudes of the mentioned mesonic decays, thus enabling one to evade these bounds. But let us recap what the current limits on the masses of the various Higgs bosons from direct searches at previous and current colliders are:

(i) LEP limits: A lower limit of $114 \mathrm{GeV}$ was imposed for both Higgs bosons, which are $C P$-even states, whether SM-like or not, albeit the lower mass region is ambiguous, given a slight excess observed at the large electron-positron (LEP) collider for an invariant mass around $98 \mathrm{GeV}$ [30]. In the minimal supersymmetric SM configuration, for large $\tan \beta$ and low mass for the $C P$-odd Higgs boson, being the lightest Higgs boson $h$ non-SM-like, the limits on the neutral masses are $m_{A}>93.4 \mathrm{GeV}$ and $m_{h}>92.8 \mathrm{GeV}$ [31]. Furthermore, from the Higgs-strahlung process, the LEP collaborations have established as lower bound for the heavy neutral Higgs bosons mass a value of $112 \mathrm{GeV}$ [32]. For the mass of the charged Higgs boson, the LEP collaborations have instead established a lower bound at $78.6 \mathrm{GeV}$ [31].

(ii) Tevatron limits: The CDF Collaboration reported a local excess in the mass region $130 \mathrm{GeV}<m_{h}<$ $160 \mathrm{GeV}$ [33], and D0 found a local fluctuation in the $H^{0}$ mass region from 110 to $125 \mathrm{GeV}$ [34]. These are consistent with the later discovery of the $125 \mathrm{GeV}$ state. For the case of a mass of the charged Higgs boson in the range 90 to $160 \mathrm{GeV}$, the $\mathrm{CDF}$ and D0 experiments extracted a limit for the $\mathrm{BR}\left(t \rightarrow H^{+} b\right)$ of approximately $20 \%$ considering both cases $\operatorname{BR}\left(H^{+} \rightarrow c \bar{s}\right)=1$ and $\operatorname{BR}\left(H^{+} \rightarrow \tau^{+} \nu\right)=$ 1 [35-37]. 
(iii) LHC limits: The almost degenerate case for the masses of Higgs bosons in the range $110 \mathrm{GeV}<$ $m_{H}<150 \mathrm{GeV}$ has been analyzed by CMS, and the experimental results can be applied in a generic way to a $C P$-odd state, too [38]. The case of additional states with exactly the same mass of the discovered Higgs boson (when it is SM-like) is not discarded, and some models could reproduce it, like the 2HDM-I [39]. In contrast, a fermiophobic Higgs boson with mass in the range 110 to $188 \mathrm{GeV}$ has been excluded by CMS [40]. Concerning $C P$-odd states, lately, the CMS Collaboration has reported a slight excess with a mass just above $97.6 \mathrm{GeV}$ [41]. However, the ATLAS experiment has not observed a corresponding significant excess [22]. Besides, recently, the CMS Collaboration has excluded small values of $\tan \beta$ in the framework of any $2 \mathrm{HDM}$ in the range $225 \mathrm{GeV}<m_{A}<1000 \mathrm{GeV}$ [42]. For the analysis of a charged Higgs boson, CMS has set $\operatorname{BR}\left(t \rightarrow H^{+} b\right)=2-3 \%$ as the upper limit for the case $\operatorname{BR}\left(H^{+} \rightarrow \tau^{+} \nu\right)=1$ in the range of masses varying from 80 to $160 \mathrm{GeV}$ [22]. Otherwise, assuming $\operatorname{BR}\left(H^{+} \rightarrow c \bar{s}\right)=1$, ATLAS and CMS set $\operatorname{BR}\left(t \rightarrow H^{+} b\right) \approx 20 \%$ for the mass range 90 to $160 \mathrm{GeV}$ [22]. Finally, quite recently, CMS set a limit of $\operatorname{BR}\left(t \rightarrow H^{+} b\right)=$ $0.5-0.8 \%$ for the case $\operatorname{BR}\left(H^{+} \rightarrow c \bar{b}\right)=1$ in the mass range 90 to $150 \mathrm{GeV}$ [43].

So, considering all experimental bounds and theoretical constraints, we proceed to choose several scenarios. Specifically, we consider four scenarios, wherein relevant benchmarks points (BPs) are defined according to the standard Yukawa prescriptions: type I (in which one Higgs doublet couples to all fermions), type II (in which one Higgs doublet couples to the up-type quarks and the other couples to the down-type quarks), type $X$ (also called IV or lepton-specific, in which the quark couplings are type I and the lepton ones are type II), and type Y (also called III or the flipped model, in which the quark couplings are type II and the lepton ones are type I).

For a light charged Higgs boson, in the 2HDM-III, the most important decay channels are $H^{-} \rightarrow s \bar{c}$ and $b \bar{c}$, when $Y \gg X, Z$ (like-I scenario), $X, Z \gg Y$ (like-II scenario), or $X \gg Y, Z$ (like-Y scenario), in which case the mode $H^{-} \rightarrow$ $b \bar{c}$ receives a substantial enhancement, coming from the four-zero texture implemented in the Yukawa matrices, so as to obtain even a $\operatorname{BR}\left(H^{-} \rightarrow b \bar{c}\right) \approx 0.95$, so that we focus on this decay, also owing to the fact that it can be $b$ tagged, thus reducing in turn the level of background. However, for the case $Z \gg X, Y$ (like- $X$ scenario), the decay channel $H^{-} \rightarrow \tau \bar{\nu}_{\tau}$ is maximized, reaching a BR of $90 \%$ or so [11], so we will investigate this mode as well.

In this work, considering the parameter scan performed in Ref. [8], we adopt the following BPs, where the two aforementioned decay channels $\left(H^{-} \rightarrow b \bar{c}\right.$ and $\left.H^{-} \rightarrow \tau \bar{\nu}_{\tau}\right)$ offer the most optimistic chances for detection:

(i) Scenario 2HDM-III like-I: $\cos (\beta-\alpha)=0.5$, $\chi_{22}^{u}=1, \chi_{23}^{u}=0.1, \chi_{33}^{u}=1.4, \chi_{22}^{d}=1.8, \chi_{23}^{d}=0.1$, $\chi_{33}^{d}=1.2, \quad \chi_{22}^{\ell}=-0.4, \quad \chi_{23}^{\ell}=0.1, \chi_{33}^{\ell}=1$ with $Y \gg X, Z$.

(ii) Scenario 2HDM-III like-II: $\cos (\beta-\alpha)=0.1$, $\chi_{22}^{u}=1, \quad \chi_{23}^{u}=-0.53, \quad \chi_{33}^{u}=1.4, \quad \chi_{22}^{d}=1.8$, $\chi_{23}^{d}=0.2, \quad \chi_{33}^{d}=1.3, \quad \chi_{22}^{\ell}=-0.4, \quad \chi_{23}^{\ell}=0.1$, $\chi_{33}^{\ell}=1$ with $X, Z \gg Y$.

(iii) Scenario $2 \mathrm{HDM}-\mathrm{III}$ like-X: the same parameters of scenario 2HDM-III like-II but $Z \gg X, Y$.

(iv) Scenario 2HDM-III like-Y: the same parameters of scenario 2HDM-III like-II but $X \gg Y, Z$.

For all four benchmarks scenarios, we assume $m_{h}=$ $125 \mathrm{GeV}$ and consider $m_{A}=100 \mathrm{GeV}, m_{H}=190 \mathrm{GeV}$, and $100 \mathrm{GeV}<m_{H^{ \pm}}<170 \mathrm{GeV}$.

Before proceeding to investigate the two aforementioned $\mathrm{H}^{-}$decays, in order to gain some insight into the inclusive event rates available, we show in Figs. 4 and 5 a scan over the relevant parameters $X, Y$, and $Z$ for the four 2HDM-III incarnations, each in correspondence of the relevant
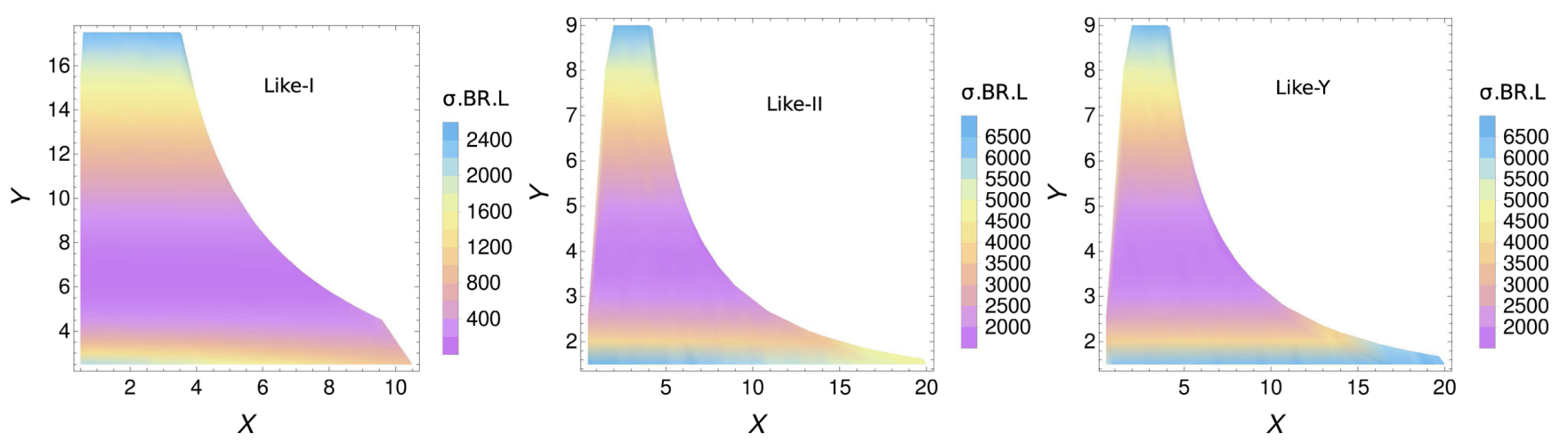

FIG. 4. Event rates $\sigma$.BR. $L$ over the $(X, Y)$ plane at the LHeC with $\sqrt{s_{e p}} \approx 1.3 \mathrm{TeV}$, where $\sigma \equiv \sigma\left(e p \rightarrow \nu_{e} H^{-} q\right)$ with $q=q_{l}$ or $b$ is the production cross section, $L=100 \mathrm{fb}^{-1}$ is the integrated luminosity, and BR is the decay fraction for the channel $H^{-} \rightarrow b \bar{c}$, for the following 2HDM-III scenarios: like-I (left), like-II (center), and like-Y (right). 


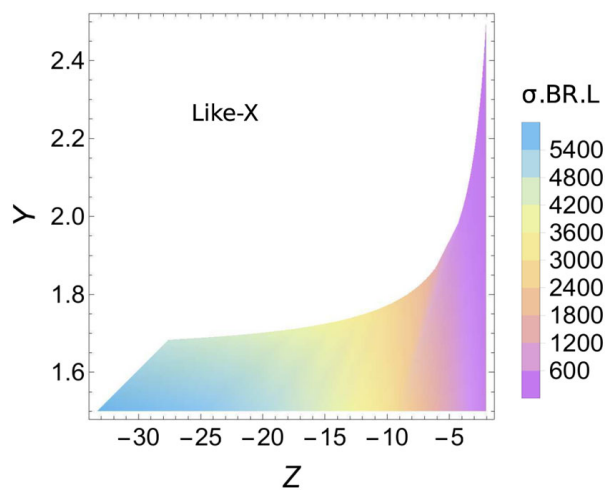

FIG. 5. Event rates $\sigma . \mathrm{BR} . L$ at the LHeC with $\sqrt{s_{e p}} \approx 1.3 \mathrm{TeV}$, where $\sigma \equiv \sigma\left(e p \rightarrow \nu_{e} H^{-} q\right)$ with $q=q_{l}$ or $b$ is the production cross section, $L=100 \mathrm{fb}^{-1}$ is the integrated luminosity, and BR is the decay fraction for the channel $H^{-} \rightarrow \tau \bar{\nu}_{\tau}$, for the following 2HDM-III scenario: like-X.

$H^{-} \rightarrow b \bar{c}$ and $H^{-} \rightarrow \tau \bar{\nu}_{\tau}$ decay channels. Assuming the LHeC standard c.m. energy of $\sqrt{s_{e p}} \approx 1.3 \mathrm{TeV}$ and a luminosity of $L=100 \mathrm{fb}^{-1}$, it is clear that inclusive event rates are substantial, of order up to several thousands in all four cases, so that the potential of the $\mathrm{LHeC}$ in extracting the $H^{-} \rightarrow b \bar{c}$ and $H^{-} \rightarrow \tau \nu_{\tau}$ decays is definitely worth exploring further. In fact, the main objective of our analysis is to tension one decay against the other and extract the corresponding significances, which may lead to evidencing or indeed discovering the true underlying $2 \mathrm{HDM}$ structure responsible for EWSB.

\section{DISCUSSION}

As intimated, in the framework of the 2HDM-III considered here, there are two main $H^{ \pm}$decay channels, which are $H^{-} \rightarrow b \bar{c}$ (the leading one for the incarnations like-I, -II, and -Y) and $H^{-} \rightarrow \tau \bar{\nu}_{\tau}$ (the leading one for the incarnation like-X). Some BPs, maximizing the signal rates in the four 2HDM-III incarnations defined in terms of the parameters $\chi_{i j}^{f}$ and $X, Y$, and $Z$ introduced previously, are given in Table II, wherein the relevant BRs of the $H^{ \pm}$state are given alongside the cross sections of the associated production process ep $\rightarrow \nu_{e} H^{-} q$, where $q=q_{l}$ or $b$. (However, we have eventually verified that only the case $q=b$ is phenomenologically relevant so that, henceforth, we neglect discussing the case $q=q_{l}$ explicitly, though it is included in our simulations.)

The signatures that we will consider are as follows.

(i) On the one hand, in connection with the 2HDM-III like-I, -II, and -Y, wherein the most relevant decay process is $H^{-} \rightarrow b \bar{c}$, the final state is $3 j+E_{T}$ (where $j$ is a generic jet and $E_{T}$ refers to missing transverse energy), with one $b$-tagged and one light jet (associated to the charged Higgs boson reconstruction) accompanied by a remaining jet, which can be $b$ tagged or not.

(ii) On the other hand, in connection with the 2HDM-III like-X, wherein the most relevant decays process is $H^{-} \rightarrow \tau \bar{\nu}_{\tau}$, the final state is $j+l+E_{T}$, where $l=e$, $\mu$ (from a leptonic $\tau$ decay), and the jet is $b$ tagged.

In this upcoming discussion, we will describe the phenomenology of these two possible processes. To carry out our numerical analysis, we have used CALCHEP3.7 [44] as parton-level event generator, interfaced to the CTEQ6L1 parton distribution functions [45], then PYTHIA6 [46] for the parton shower, hadronization, and hadron decays and the pretty good simulator (PGS) package [47] as a detector emulator, by using a LHC parameter card suitably modified for the LHeC [16,17]. In particular, the detector parameters simulated were as follows: we considered a calorimeter coverage $|\eta|<5.0$, with segmentation $\Delta \eta \times \Delta \phi=$ $0.0359 \times 0314$ (the numbers of divisions in $\eta$ and $\phi$ are 320 and 200, respectively). Moreover, we used Gaussian energy resolution, with

$$
\frac{\Delta E}{E}=\frac{a}{\sqrt{E}} \oplus b
$$

where $a=0.085$ and $b=0.003$ for the electromagnetic calorimeter resolution and $a=0.32, b=0.086$ for the hadronic calorimeter resolution, with $\oplus$ meaning addition in quadrature. Herein, the values of $a$ and $b$ are parameters established by the design of the LHeC [16,17]. The algorithm to perform jet finding was a"cone" one with jet radius $\Delta R=0.5$. The calorimeter trigger cluster finding a seed (shoulder) threshold was $5 \mathrm{GeV}(1 \mathrm{GeV})$. We took $E_{T}(j)>10 \mathrm{GeV}$ for a jet to be considered so, in addition to the isolation criterion, $\Delta R(j ; l)>0.5$. Finally, we have mapped the kinematic behavior of the final-state particles using MADANALYSIS5 [48].

TABLE II. The BPs that we studied for the 2HDM-III in the incarnations like-I, -II, -X, and -Y. We present cross sections and BRs at parton level, for some $H^{ \pm}$mass choices.

\begin{tabular}{|c|c|c|c|c|c|c|c|c|c|}
\hline \multirow{2}{*}{$\begin{array}{l}\text { 2HDM-III } \\
\text { like- }\end{array}$} & \multicolumn{3}{|c|}{ Parameters } & \multicolumn{4}{|c|}{$\sigma\left(e p \rightarrow \nu_{e} H^{-} q\right)(\mathrm{pb})$} & \multirow{2}{*}{$\begin{array}{c}\mathrm{BR}\left(H^{-} \rightarrow b \bar{c}\right) \\
m_{H^{ \pm}}=110 \mathrm{GeV}\end{array}$} & \multirow{2}{*}{$\begin{array}{c}\mathrm{BR}\left(H^{-} \rightarrow \tau \bar{\nu}_{\tau}\right) \\
m_{H^{ \pm}}=110 \mathrm{GeV}\end{array}$} \\
\hline & $X$ & $Y$ & $Z$ & $m_{H^{ \pm}}=110 \mathrm{GeV}$ & $130 \mathrm{GeV}$ & $150 \mathrm{GeV}$ & $170 \mathrm{GeV}$ & & \\
\hline I & 0.5 & 17.5 & 0.5 & $2.56 \times 10^{-2}$ & $1.30 \times 10^{-2}$ & $3.47 \times 10^{-3}$ & $1.35 \times 10^{-4}$ & $9.57 \times 10^{-1}$ & $2.5 \times 10^{-4}$ \\
\hline II & 20 & 1.5 & 20 & $2.18 \times 10^{-2}$ & $1.13 \times 10^{-2}$ & $2.95 \times 10^{-3}$ & $5.89 \times 10^{-5}$ & $9.9 \times 10^{-1}$ & $2.22 \times 10^{-4}$ \\
\hline $\mathrm{X}$ & 0.03 & 1.5 & -33.33 & $6.49 \times 10^{-2}$ & $3.39 \times 10^{-2}$ & $8.83 \times 10^{-3}$ & $2.34 \times 10^{-4}$ & $9.28 \times 10^{-2}$ & $9.04 \times 10^{-1}$ \\
\hline Y & 13 & 1.5 & $-1 / 13$ & $6.41 \times 10^{-2}$ & $3.27 \times 10^{-2}$ & $8.47 \times 10^{-3}$ & $2.2 \times 10^{-4}$ & $9.91 \times 10^{-1}$ & $6.12 \times 10^{-3}$ \\
\hline
\end{tabular}



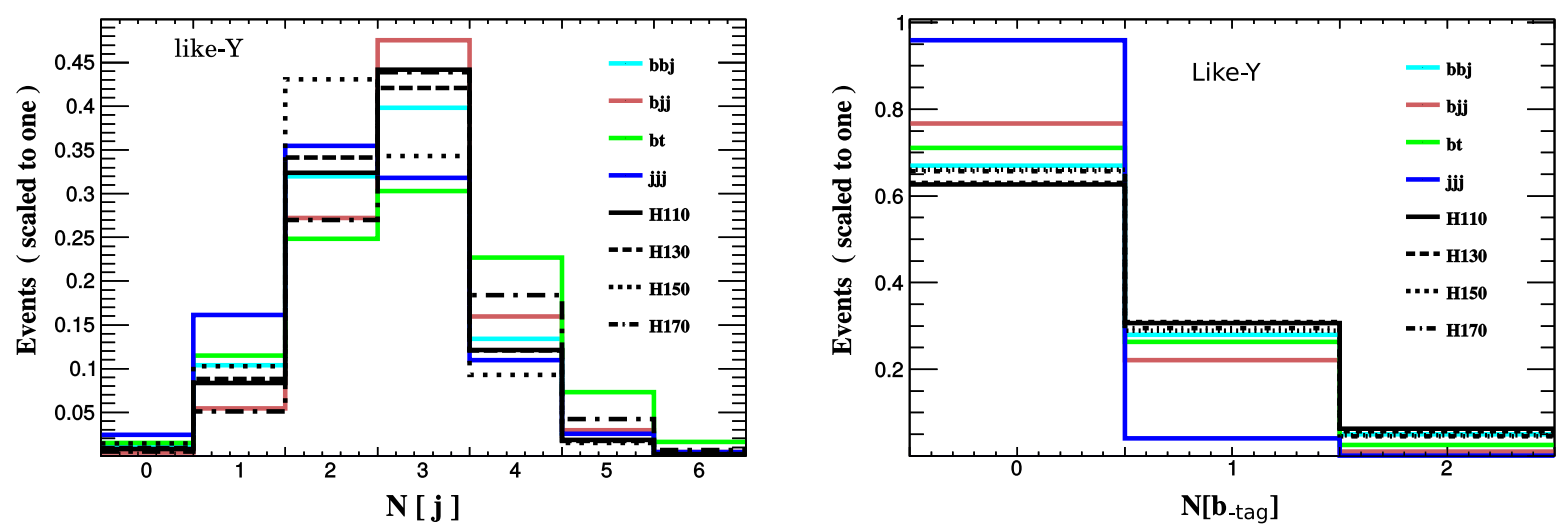

FIG. 6. Distributions for the process $e^{-} q \rightarrow \nu_{e} H^{-} b$ followed by $H^{-} \rightarrow b \bar{c}$ : in the left panel, we present the multiplicity of all jets, while in the right panel, we present the multiplicity of the $b$-tagged ones. The like-Y case is illustrated. The normalization is to unity.

\section{A. Process $e^{-} q \rightarrow \nu_{e} H^{-} b$ with $H^{-} \rightarrow b \bar{c}$ for the 2HDM-III like-I, -II, and -Y}

In this subsection, we discuss the final state with one $b$-tagged jet and one light jet (associated with the secondary decay $H^{-} \rightarrow b \bar{c}$ ) alongside a generic (i.e., light or $b$-tagged) forward jet (associated with the primary collision) plus missing transverse energy. For this case, we apply the following cuts ${ }^{3}$ :

(I) First, we select only events with exactly three jets in the final state. Then, we reject all events without a $b$-tagged jet. Hence, at this point, we keep events like $3 j+E_{T}$ with at least one $b$-tagged jet (see the histograms in Fig. 6). For these selections, our signal generally has an efficiency of $12 \%$, while the most efficient background $\nu_{e} b b j$ has a $10 \%$ response. The remaining backgrounds have efficiencies of $5 \%, 8 \%$, and $1 \%$ for $\nu_{e} b t, \nu_{e} b j j$, and $\nu_{e} j j j$, respectively.

(II) The second set of cuts is focused on selecting two jets (one $b$-tagged, labeled as $b_{\text {tag }}$, and one not, labeled as $j_{\mathrm{c}}$ ), which are central in the detector. First, we demand that $P_{T}\left(b_{\text {tag }}\right)>30(40) \mathrm{GeV}$ and $P_{T}\left(j_{\mathrm{c}}\right)>20(30) \mathrm{GeV}$ for $m_{H^{ \pm}}=110$, $130(150,170) \mathrm{GeV}$ (here, $P_{T}$ is the transverse momentum). Then, we impose a cut on the pseudorapidity $\left|\eta\left(b_{\text {tag }}, j_{\mathrm{c}}\right)\right|<2.5$ of both these jets and, finally, select events in which $1.8(2)<$ $\Delta R\left(j_{\mathrm{c}} ; b_{\mathrm{tag}}\right)<3.4(3.4)$ in correspondence of $m_{H^{ \pm}}=110,130(150,170) \mathrm{GeV}$ (where $\Delta R$ is the standard cone separation). Upon enforcing these cuts, we find that our signal has a cumulative efficiency of $7.3 \%$. The most efficient background $\nu_{e} b j j$ has a rate of $6 \%$, while the others show efficiencies of $3.3 \%, 3.7 \%$, and $0.3 \%$ (for $\nu_{e} b t$,

\footnotetext{
${ }^{3}$ For illustration, we assume the $2 \mathrm{HDM}-\mathrm{III}$ like-Y scenario in our description, though the signal kinematics is essentially independent of the theoretical setup, as it primarily depends on the $m_{H^{ \pm}}$value.
}

$\nu_{e} b b j$, and $\nu_{e} j j j$, respectively). This information is easily drawn from Fig. 7.

(III) The next cut is related to the selection of a forward third generic jet (it can be either a light jet or a $b$-tagged one). Our selection for such a third jet is $|\eta|>0.6$ (with a transverse momentum above $20 \mathrm{GeV}$ ). With this cut, our signal shows an efficiency of $5.4 \%$ while $4.2 \%$ is the rate for the most efficient background $\left(\nu_{e} b j j\right)$. The rest of the backgrounds show efficiencies below $2 \%$ for $\nu_{e} b b j$ and $\nu_{e} b t$ or $0.3 \%$ for $\nu_{e} j j j$.

(IV) The selection of the jet pair representing a $H^{ \pm}$ candidate is made by considering only events for which the invariant mass of the two central jets is in the vicinity of the (trial) mass of the charged Higgs boson. However, it must be considered that, at the detector level, the signal may see a mass shift due to the finite efficiency in selecting the wanted jet dynamics. Therefore, in the histograms of Fig. 8, we study such invariant mass in the case of our signal for, e.g., $m_{H^{ \pm}}=110$ (left) and 130 (right) $\mathrm{GeV}$. We benchmark these against the corresponding spectra from the backgrounds. From this plot, we can indeed see a shift of the signal peaks towards lower invariant masses, so that we can implement the following selection criterium: $m_{H^{ \pm}}-20 \mathrm{GeV}<$ $M\left(b_{\text {tag }}, j_{\mathrm{c}}\right)<m_{H^{ \pm}}$. Furthermore, we noticed that the invariant mass formed by the light central jet and the generic forward jet (not shown here) has a structure in most of the backgrounds, dictated by the presence of a hadronic $W^{ \pm}$boson decay. Because our signal does not have this feature, we further impose that $M\left(j_{\mathrm{c}}, j_{\mathrm{f}}\right)>80 \mathrm{GeV}$ or $M\left(j_{\mathrm{c}}, j_{\mathrm{f}}\right)<$ $60 \mathrm{GeV}$ (where $j_{\mathrm{f}}$ labels the forward jet). This combination of mass cuts is highly selective, giving us an overall efficiency of $2.4 \%$ for the signal and (at most) $0.6 \%$ for the backgrounds.

The final results, following the application of cuts I-IV, are found in Table III, for the 2HDM-III like-I, -II, and -Y 

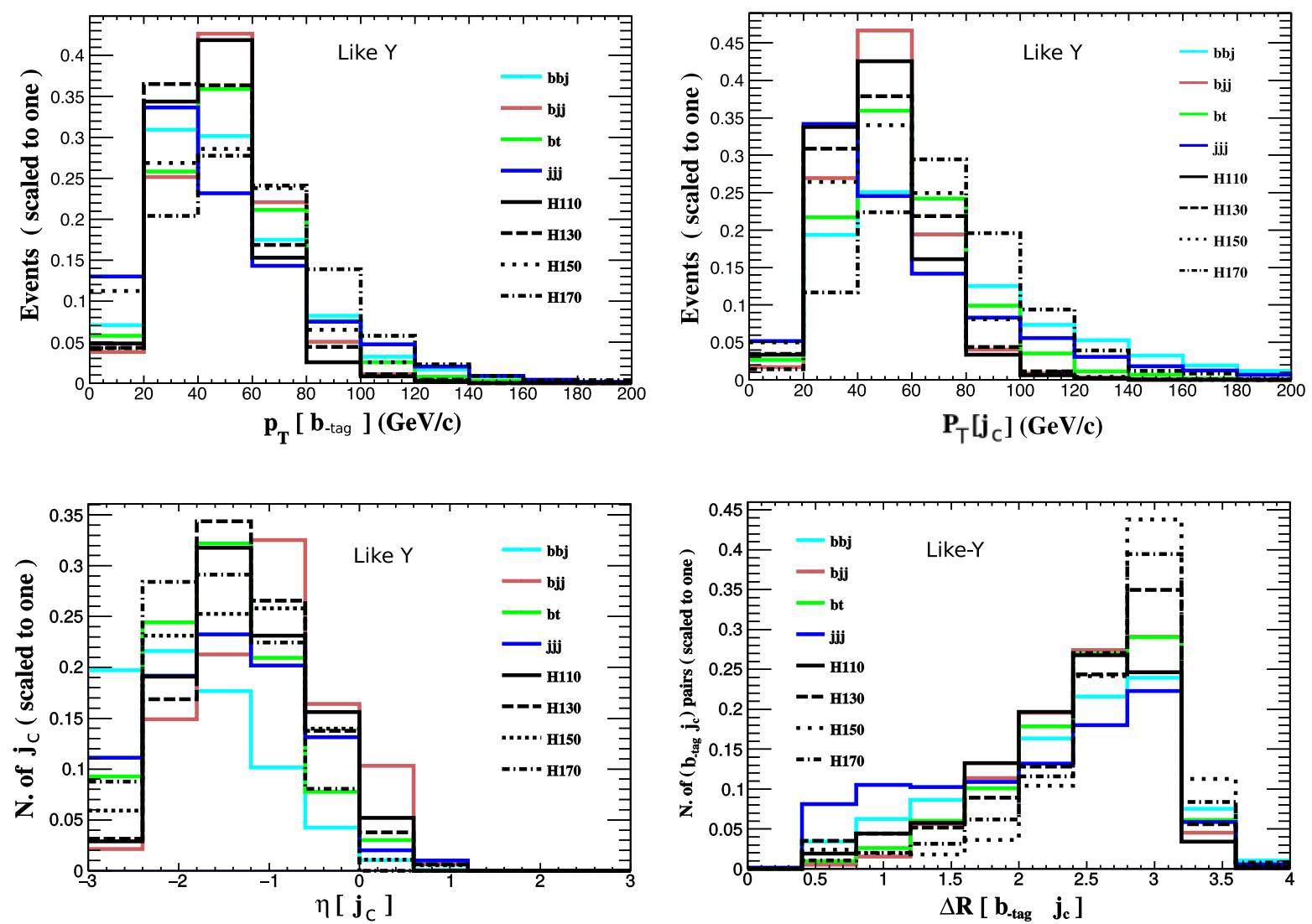

FIG. 7. Distributions for the process $e^{-} q \rightarrow \nu_{e} H^{-} b$ followed by $H^{-} \rightarrow b \bar{c}$ : in the top-left panel, we present the transverse momentum of the central $b$-tagged jet; in the top-right panel, we present the transverse momentum of the central light jet; in the bottom-left panel, we present the pseudorapidity of the central light jet; and in the bottom-right panel, we present the separation between the two central jets. The like-Y case is illustrated. The normalization is to unity.
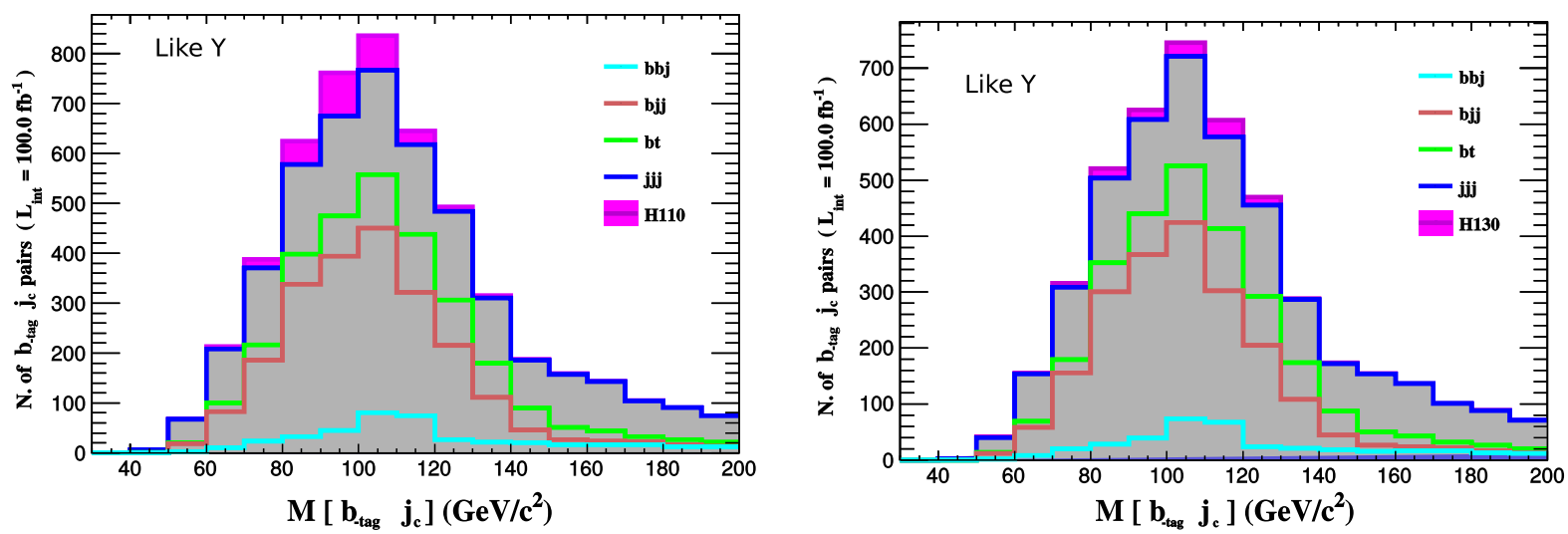

FIG. 8. Distributions for the process $e^{-} q \rightarrow \nu_{e} H^{-} b$ followed by $H^{-} \rightarrow b \bar{c}$ in the invariant mass of the two central jets for $m_{H^{ \pm}}=$ $110 \mathrm{GeV}$ (left) and $m_{H^{ \pm}}=130 \mathrm{GeV}$ (right). The like-Y case is illustrated. The normalization is to the total event rate for $L=100 \mathrm{fb}{ }^{-1}$.

incarnations. Statistically, significances of the signal $\mathcal{S}$ over the cumulative background $\mathcal{B}$ are very good at low $H^{ \pm}$ masses already for $100 \mathrm{fb}^{-1}$ of luminosity. As the latter increases, larger masses can be afforded through evidence or discovery, particularly so in the like-Y scenario. However, an ultimate mass reach is probably $130 \mathrm{GeV}$ in all cases.

\section{B. Process $e^{-} q \rightarrow \nu_{e} H^{-} b$ with $H^{-} \rightarrow \tau \bar{\nu}_{\tau}$ in the 2HDM-III like-X}

Now, we focus our attention on the channel $H^{-} \rightarrow \tau \bar{\nu}_{\tau}$. To this effect, as previously mentioned, we look at leptonic $\tau$ decays $\left(\tau \rightarrow l \bar{\nu}_{l} \nu_{\tau}\right.$, with $\left.l=e, \mu\right)$, and we $b$ tag the prompt (i.e., coming from the primary collision) jet in the final state. The cuts to extract our signal are presented below: 
TABLE III. Significances obtained after the sequential cuts described in the text for the signal process $e^{-} q \rightarrow \nu_{e} H^{-} b$ followed by $H^{-} \rightarrow b \bar{c}$ for four BPs in the 2HDM-III like-I, -II, and -Y. The simulation is done at detector level. In the column scenario, the label A$110(130)[150]\{170\}$ means $m_{H^{ \pm}}=110(130)[150]\{170\} \mathrm{GeV}$ in the 2HDM-III like-A, where A can be I, II, and Y.

\begin{tabular}{|c|c|c|c|c|c|c|c|}
\hline Signal & Scenario & Events (raw) & Cut I & Cut II & Cut III & Cut IV & $(S / \sqrt{B})_{100 \mathrm{fb}^{-1}\left(1000 \mathrm{fb}^{-1}\right)\left[3000 \mathrm{fb}^{-1}\right]}$ \\
\hline \multirow[t]{4}{*}{$\nu_{e} H^{ \pm} b$} & $\mathrm{I}-110$ & 2562 & 298 & 182 & 134 & 54 & $1.43(4.52)[7.82]$ \\
\hline & $\mathrm{I}-130$ & 1300 & 139 & 82 & 64 & 19 & $0.58(1.82)[3.16]$ \\
\hline & $\mathrm{I}-150$ & 347 & 29 & 13 & 11 & 3 & $0.16(0.5)[0.86]$ \\
\hline & $\mathrm{I}-170$ & 13 & 1.29 & 0.62 & 0.51 & 0.14 & $0.01(0.03)[0.05]$ \\
\hline \multirow[t]{4}{*}{$\nu_{e} H^{ \pm} b$} & II-110 & 2183 & 245 & 151 & 122 & 53 & $1.4(4.43)[7.68]$ \\
\hline & II-130 & 1128 & 128 & 84 & 71 & 22 & $0.7(2.21)[3.82]$ \\
\hline & II- 150 & 294 & 28 & 14 & 13 & 4 & $0.2(0.65)[1.13]$ \\
\hline & II-170 & 6 & 0.6 & 0.33 & 0.3 & 0.08 & $0.005(0.017)[0.029]$ \\
\hline \multirow[t]{4}{*}{$\nu_{e} H^{ \pm} b$} & Y-110 & 6417 & 468 & 567 & 347 & 156 & 4.18 (12.99) [22.5] \\
\hline & Y-130 & 3268 & 366 & 204 & 156 & 46 & $1.43(4.53)[7.84]$ \\
\hline & Y-150 & 847 & 68 & 29 & 23 & 6 & 0.33 (1.06) [1.83] \\
\hline & Y-170 & 22 & 2.3 & 1.12 & 0.89 & 0.25 & $0.017(0.05)$ [0.09] \\
\hline$\nu_{e} b b j$ & & 20169 & 2011 & 748 & 569 & 125 & \multirow{4}{*}{$\begin{array}{c}\mathcal{B}=1441 \\
\sqrt{\mathcal{B}}=37.9\end{array}$} \\
\hline$\nu_{e} b j j$ & & 117560 & 10278 & 7211 & 5011 & 718 & \\
\hline$\nu_{e} b t$ & & 41885 & 2278 & 1418 & 1130 & 188 & \\
\hline$\underline{\nu_{e} j j j}$ & & 867000 & 9238 & 3221 & 2593 & 409 & \\
\hline
\end{tabular}

(I) This first set of cuts is focused on selecting events with one $b$-tagged jet and one lepton, by imposing $\left|\eta\left(b_{\text {tag }}, l\right)\right|<2.5, P_{T}\left(b_{\text {tag }}, l\right)>20 \mathrm{GeV}$ and the isolation condition $\Delta R\left(b_{\mathrm{tag}} ; l\right)>0.5$ (see Fig. 9 for the histograms of the lepton and jet multiplicities.) Following this, we find that our signal has an efficiency of $14 \%$, whereas the backgrounds $\nu_{e} \nu_{l} l j$ and $\nu_{e} \nu_{l} l b$ have rates of $23 \%$ and $18 \%$, respectively. The remaining noise shows an efficiency below $5 \%$.

(II) The next set of cuts enables us to select a stiffer lepton and impose conditions on the missing transverse energy, which are adapted to the trial $H^{ \pm}$mass. We select events with $P_{T}(l)>25(40) \mathrm{GeV}$ and $E_{T}>30(40) \mathrm{GeV}$ for $m_{H^{ \pm}}=110,130(150,170) \mathrm{GeV}$. Our signal presents an efficiency of $70 \%$, while $80 \%$ is

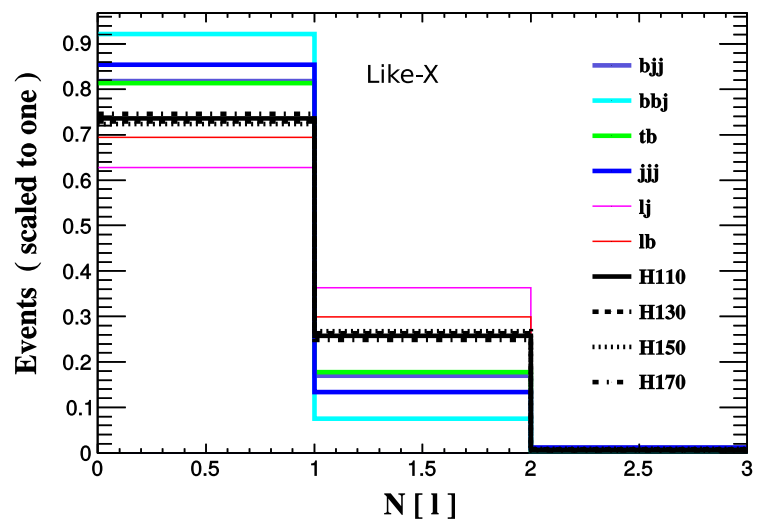

the rate for $\nu_{e} \nu_{l} l j, \nu_{e} \nu_{l} l b$, and $\nu_{e} t b$. The remaining backgrounds show efficiencies of $60 \%$ or below (see Fig. 10).

(III) Then, based on the left frame of Fig. 11, we require $\left|\eta\left(b_{\text {tag }}\right)\right|>0.5$. Furthermore, upon defining the total hadronic transverse energy $H_{T}=\sum_{\text {hadronic }}\left|P_{T}\right|$ in the final state, based on the right frame of Fig. 11, we select $H_{T}<60 \mathrm{GeV}$. For our signal, these cuts are a little discriminatory and show an efficiency of $75 \%$. However, for all backgrounds, the efficiency is in general below $50 \%$.

(IV) Finally, we enforce the last selection by exploiting the transverse mass $M_{T}(l)^{2}=$ $2 p_{T}(l) E_{T} /(1-\cos \phi)$, where $\phi$ is the relative azimuthal angle between $p_{T}(l)$ and $E_{T}$, a quantity which allows one to label the candidate events

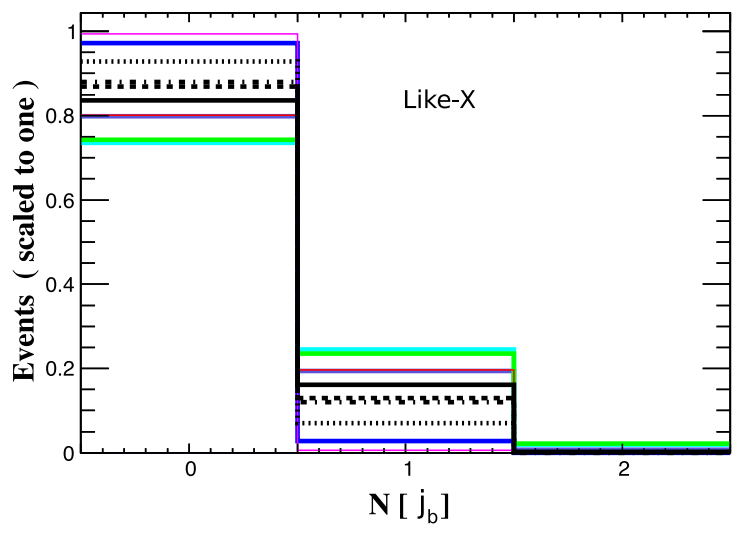

FIG. 9. Distributions for the process $e^{-} q \rightarrow \nu_{e} H^{-} b$ followed by $H^{-} \rightarrow \tau \bar{\nu}_{\tau}^{-}$: in the left(right) panel, we present the number of leptons ( $b$ jets) per event. The like- $\mathrm{X}$ case is illustrated. The normalization is to unity. 

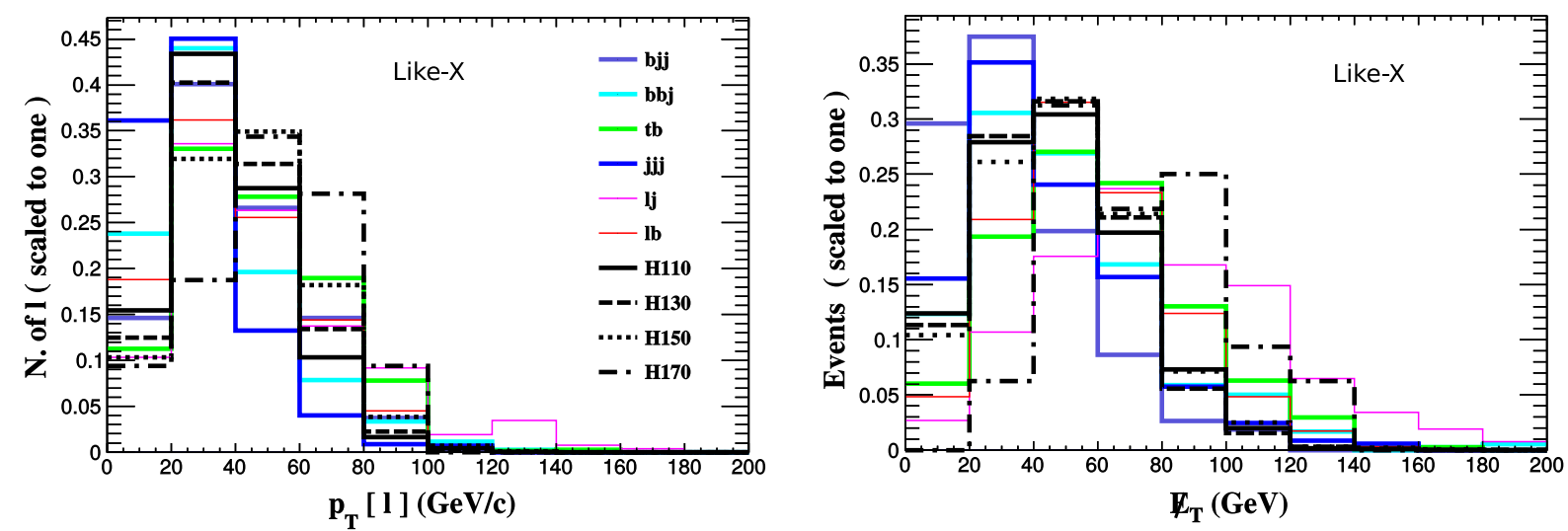

FIG. 10. Distributions for the process $e^{-} q \rightarrow \nu_{e} H^{-} b$ followed by $H^{-} \rightarrow \tau \bar{\nu}_{\tau}$ : in the left panel, we present the transverse momentum of the lepton, while in the right panel, we present the total missing transverse energy. The like-X case is illustrated. The normalization is to unity.
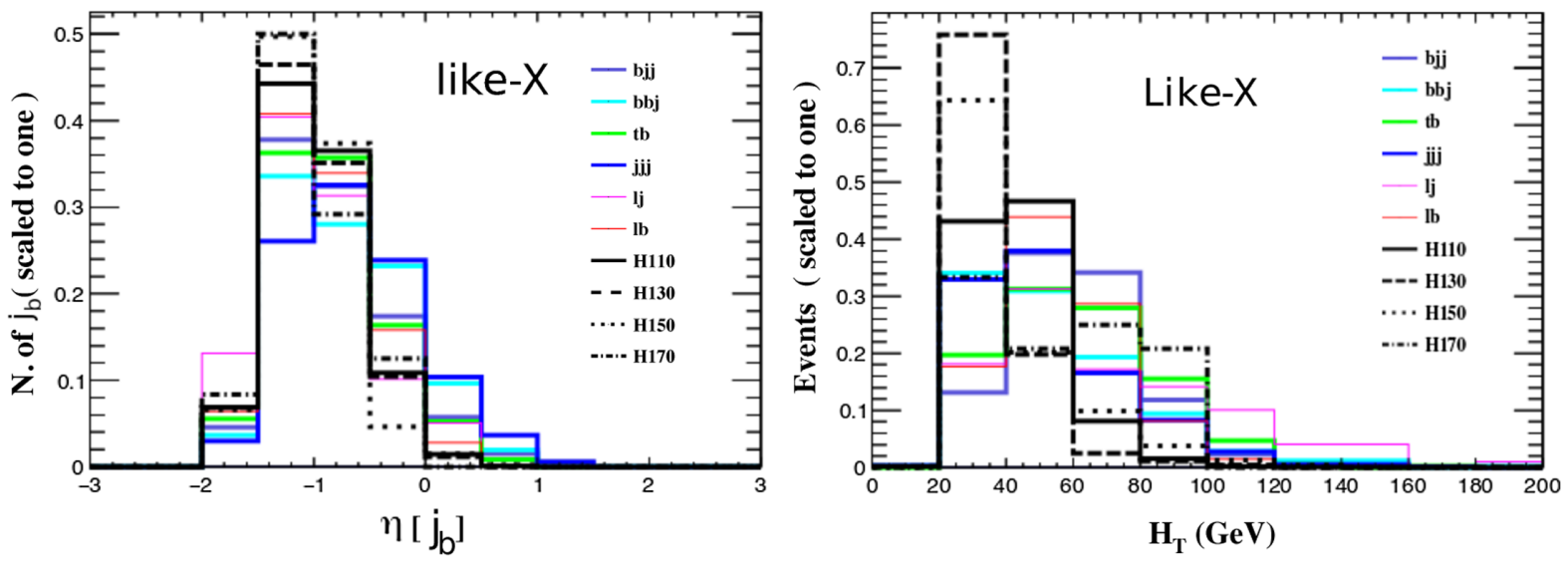

FIG. 11. Distributions for the process $e^{-} q \rightarrow \nu_{e} H^{-} b$ followed by $H^{-} \rightarrow \tau \bar{\nu}_{\tau}$ : in the left panel, we present the pseudorapidity of the $b$ jet, while in the right panel, we present the total hadronic transverse energy. The like-X case is illustrated. The normalization is to unity.
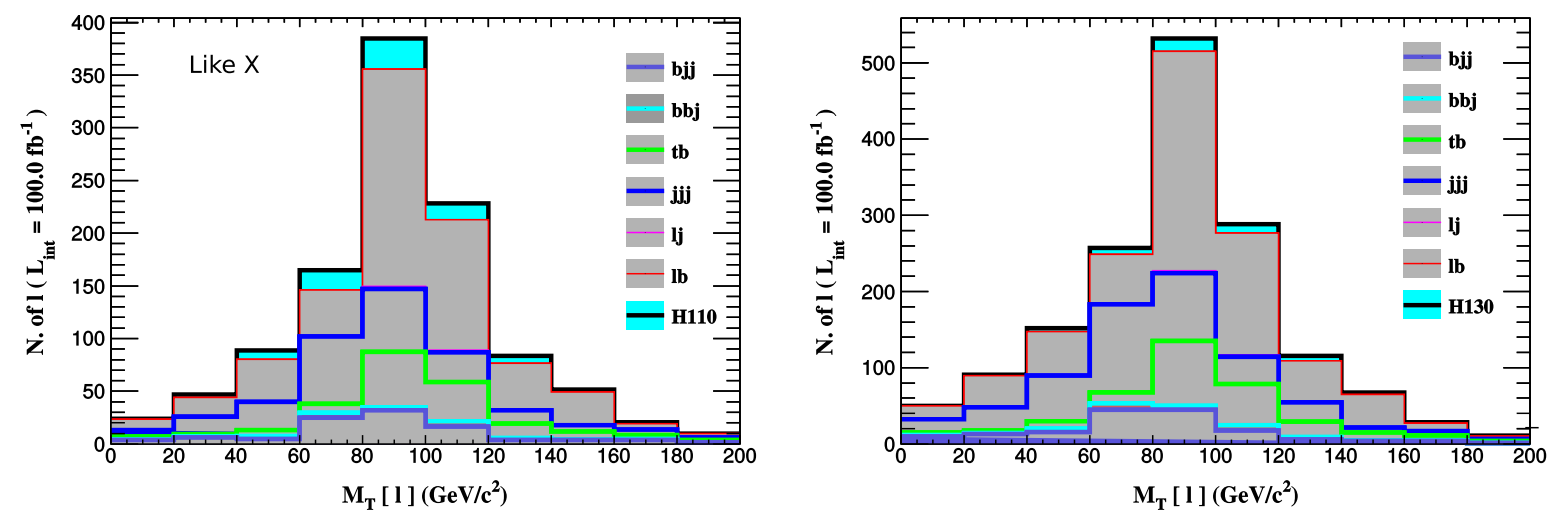

FIG. 12. Distributions for the process $e^{-} q \rightarrow \nu_{e} H^{-} b$ followed by $H^{-} \rightarrow \tau \bar{\nu}_{\tau}$ in the transverse mass of the final state for $m_{H^{ \pm}}=$ $110 \mathrm{GeV}$ (left) and $m_{H^{ \pm}}=130 \mathrm{GeV}$ (right). The like-X case is illustrated. The normalization is to the total event rate for $L=100 \mathrm{fb}{ }^{-1}$.

reconstructing the charged Higgs boson mass. However, the existence of one additional neutrino in the final state $\left(\nu_{e}\right)$ emerging from the primary hard collision, alongside the two stemming from the $\tau$ decay $\left(\nu_{\tau}\right.$ and $\left.\nu_{l}\right)$, generates a widening of the transverse mass distribution of the signal. Therefore, we make the following selection: $m_{H^{ \pm}}-50 \mathrm{GeV}<$ $M_{T}(l)<m_{H^{ \pm}}+10 \mathrm{GeV}$ (see Fig. 12). For this cut, 
TABLE IV. Significances obtained after the sequential cuts described in the text for the signal process $e^{-} q \rightarrow \nu_{e} H^{-} b$ followed by $H^{-} \rightarrow \tau \bar{\nu}_{\tau}$ for four BPs in the 2HDM-III like-X. The simulation is done at detector level. In the column "Scenario", the label X $110(130)[150]\{170\}$ means $m_{H^{ \pm}}=110(130)[150]\{170\} \mathrm{GeV}$ in the 2HDM-III like-X.

\begin{tabular}{|c|c|c|c|c|c|c|c|}
\hline Signal & Scenario & Events (raw) & Cut I & Cut II & Cut III & Cut IV & $(S / \sqrt{B})_{100 \mathrm{fb}^{-1}\left(1000 \mathrm{fb}^{-1}\right)\left[3000 \mathrm{fb}^{-1}\right]}$ \\
\hline \multirow[t]{4}{*}{$\nu_{e} H^{-} q$} & $\mathrm{X}-110$ & 6480 & 178 & 124 & 94 & 67 & $2.41(7.61)[13.19]$ \\
\hline & $X-130$ & 3390 & 75 & 54 & 52 & 35 & $1.13(3.58)[6.2]$ \\
\hline & $X-150$ & 880 & 6 & 3 & 2 & 2 & $0.09(0.29)[0.5]$ \\
\hline & $X-170$ & 20 & 0.4 & 0.3 & 0.2 & 0.09 & $0.01(0.02)[0.04]$ \\
\hline$\nu_{e} b b j$ & & 20170 & 85 & 56 & 23 & 13 & \\
\hline$\nu_{e} b j j$ & & 117559 & 623 & 340 & 122 & 84 & \\
\hline$\nu_{e} t b$ & & 48845 & 460 & 374 & 149 & 105 & $\mathcal{B}=763$ \\
\hline$\nu_{e} j j j$ & & 867000 & 981 & 596 & 267 & 162 & $\sqrt{\mathcal{B}}=27.62$ \\
\hline$\nu_{e} l \nu_{l} j$ & & 23700 & 29 & 26 & 8 & 5 & \\
\hline$\nu_{e} l \nu_{l} b$ & & 40400 & 1500 & 1203 & 569 & 392 & \\
\hline
\end{tabular}

our signal has a cumulative efficiency of $1 \%$, quite comparable to the efficiency of $\nu l b$, which is $0.9 \%$. The rest of the backgrounds are instead below $0.2 \%$. The effectiveness of this selection strategy is confirmed by the final results in Table IV, wherein we present the signal and background rates along with the corresponding significances after cuts I-IV for the usual values of luminosity. Again, also in the like-X case, good sensitivity exists up to $H^{ \pm}$masses of $130 \mathrm{GeV}$.

\section{CONCLUSIONS}

In conclusion, we have assessed the potential of a possible future $\mathrm{LHeC}$, obtained from crossing $e^{-}$and $p$ beams in the CERN tunnel currently hosting the LHC and previously LEP. The foreseen beam energies are $60 \mathrm{GeV}$ and $7 \mathrm{TeV}$, respectively. Such an environment is rather clean and, since it primarily relies on a charged $W^{-}$current for the hard scattering, conducive to the production of a negatively charged Higgs boson, $H^{-}$. This state is typical of 2HDMs, and it is notoriously elusive at the LHC $[13,49]$, so it is natural to assess the scope for its detection at the $\mathrm{LHeC}$. For our 2HDM theoretical framework we have adopted a 2HDM-III supplemented by a four-zero texture in the Yukawa sector which enables one, first, to avoid imposing a $Z_{2}$ symmetry to prevent FCNCs and, second, to recreate the standard 2HDM setups, known as type I, II, X, and Y, through suitable choices of the texture matrix elements. Such a scenario can realistically only afford one with $\mathrm{LHeC}$ sensitivity to rather light $H^{ \pm}$masses, i.e., well below the top mass. In this mass regime, though, we have established that the $\mathrm{LHeC}$ can access $H^{ \pm}$masses up to $130 \mathrm{GeV}$ or so, for luminosity conditions already foreseen for such a machine. This assessment is essentially similar for all 2HDM-III incarnations, although sensitivity is primarily established in the like-I, -II, and -Y cases via $H^{-} \rightarrow b \bar{c}$ and in the like-X case via $H^{-} \rightarrow \tau \bar{\nu}_{\tau}$ (assuming electron/muon decays of the $\tau$ ). The LHeC production mode is $e^{-} q \rightarrow \nu_{e} H^{-} q$, with $q=b$ being the dominant subchannel, the latter being also induced by neutral Higgs boson exchange in $t$ channel (see Fig. 1). Hence, on the one hand, one can exploit the very efficient $b$ tagging expected at the $\mathrm{LHeC}$ detectors in order to establish the two signals above and beyond a variety of background channels, which we have done here, while, on the other hand, one could attempt to extract the $\phi_{i}^{0} W^{+} H^{-}\left(\phi_{i}^{0}=h, H, A\right)$ vertex "directly" in LHeC production (unlike the LHC, where it can only be done "indirectly" in $H^{-}$decays), which is what we shall do in a future publication.

\section{ACKNOWLEDGMENTS}

S. M. is financed in part through the NExT Institute. S.M. also acknowledges support from the UK STFC Consolidated Grant No. ST/L000296/1 and the H2020-MSCA-RISE-2014 Grant No. 645722 (NonMinimalHiggs). S. R.-N. thanks the Southampton High Energy Physics Group for hospitality while parts of this work were completed. J. H.-S. and C. H. have been supported by SNI-CONACYT (Mexico), VIEP-BUAP, and PRODEP-SEP (Mexico) under the grant "Red Temática: Física del Higgs y del Sabor." S. R. N. acknowledges a scholarship from CONACYT (Mexico). We all acknowledge useful discussions with Siba Prasad Das. 
[1] G. Aad et al. (ATLAS Collaboration), Phys. Lett. B 716, 1 (2012).

[2] S. Chatchyan et al. (CMS Colllaboration), Phys. Lett. B 716, 30 (2012).

[3] G. C. Branco, P. M. Ferreira, L. Lavoura, M. N. Rebelo, M. Sher, and J. P. Silva, Phys. Rep. 516, 1 (2012).

[4] H. Fritzsch and Z.z. Xing, Phys. Lett. B 555, 63 (2003).

[5] J. L. Diaz-Cruz, J. Hernandez-Sanchez, S. Moretti, R. Noriega-Papaqui, and A. Rosado, Phys. Rev. D 79, 095025 (2009).

[6] J. Hernandez-Sanchez, O. Flores-Sanchez, C. G. Honorato, S. Moretti, and S. Rosado, Proc. Sci., CHARGED2016 (2017) 032.

[7] J. Hernandez-Sanchez, S. P. Das, S. Moretti, A. Rosado, and R. Xoxocotzi-Aguilar, Proc. Sci., DIS2015 (2015) 227.

[8] S. P. Das, J. Hernandez-Sanchez, S. Moretti, A. Rosado, and R. Xoxocotzi, Phys. Rev. D 94, 055003 (2016).

[9] A. Cordero-Cid, J. Hernandez-Sanchez, C. G. Honorato, S. Moretti, M. A. Perez, and A. Rosado, J. High Energy Phys. 07 (2014) 057.

[10] O. Felix-Beltran, F. Gonzalez-Canales, J. HernandezSanchez, S. Moretti, R. Noriega-Papaqui, and A. Rosado, Phys. Lett. B 742, 347 (2015).

[11] J. Hernandez-Sanchez, S. Moretti, R. Noriega-Papaqui, and A. Rosado, J. High Energy Phys. 07 (2013) 044.

[12] J. Hernandez-Sanchez, S. Moretti, R. Noriega-Papaqui, and A. Rosado, Proc. Sci., CHARGED2012 (2012) 029.

[13] A. G. Akeroyd et al., Eur. Phys. J. C 77, 276 (2017).

[14] A. G. Akeroyd, S. Moretti, and J. Hernandez-Sanchez, Phys. Rev. D 85, 115002 (2012).

[15] A. M. Sirunyan et al. (CMS Collaboration), Phys. Rev. Lett. 121, 121801 (2018).

[16] J. L. Abelleira Fernandez et al. (LHeC Study Group), J. Phys. G 39, 075001 (2012).

[17] O. Bruening and M. Klein, Mod. Phys. Lett. A 28, 1330011 (2013).

[18] J. F. Gunion and H. E. Haber, Phys. Rev. D 67, 075019 (2003).

[19] J.-M. Gerard and M. Herquet, Phys. Rev. Lett. 98, 251802 (2007).

[20] S. de Visscher, J. M. Gerard, M. Herquet, V. Lemaitre, and F. Maltoni, J. High Energy Phys. 08 (2009) 042.

[21] S. Kanemura, Y. Okada, H. Taniguchi, and K. Tsumura, Phys. Lett. B 704, 303 (2011).

[22] M. Tanabashi et al. (Particle Data Group), Phys. Rev. D 98, 030001 (2018).

[23] G. Aad et al. (ATLAS and CMS Collaborations), J. High Energy Phys. 08 (2016) 045.

[24] M. Aaboud et al. (ATLAS Collaboration), Phys. Lett. B 784, 345 (2018).

[25] M. Aaboud et al. (ATLAS Collaboration), Phys. Lett. B 786, 114 (2018).
[26] M. Aaboud et al. (ATLAS Collaboration), J. High Energy Phys. 10 (2017) 112.

[27] A. M. Sirunyan et al. (CMS Collaboration), arXiv: 1809.10733.

[28] A. M. Sirunyan et al. (CMS Collaboration), J. High Energy Phys. 11 (2018) 152.

[29] A. Crivellin, C. Greub, and A. Kokulu, Phys. Rev. D 87, 094031 (2013).

[30] R. Barate et al. (ALEPH, DELPHI, L3, and OPAL Collaborations and LEP Working Group for Higgs boson searches), Phys. Lett. B 565, 61 (2003).

[31] S. Schael et al. (ALEPH, DELPHI, L3, and OPAL Collaborations and LEP Working Group for Higgs Boson Searches), Eur. Phys. J. C 47, 547 (2006).

[32] M. M. Kado and C. G. Tully, Annu. Rev. Nucl. Part. Sci. 52, 65 (2002).

[33] T. Aaltonen et al. (CDF Collaboration), Phys. Rev. D 85, 032005 (2012).

[34] V. M. Abazov et al. (D0 Collaboration), Phys. Lett. B 698, 97 (2011).

[35] B. Abbott et al. (D0 Collaboration), Phys. Rev. Lett. 82, 4975 (1999).

[36] A. Abulencia et al. (CDF Collaboration), Phys. Rev. Lett. 96, 042003 (2006).

[37] V. M. Abazov et al. (D0 Collaboration), Phys. Lett. B 682, 278 (2009).

[38] CMS Collaboration, Report No. CMS-PAS-HIG-13-016.

[39] S. Heinemeyer et al. (LHC Higgs Cross Section Working Group), arXiv:1307.1347.

[40] S. Chatrchyan et al. (CMS Collaboration), Phys. Lett. B 725, 36 (2013).

[41] CMS Collaboration, Report No. CMS-PAS-HIG-17-013.

[42] A. M. Sirunyan et al. (CMS Collaboration), arXiv: 1903.00941.

[43] A. M. Sirunyan et al. (CMS Collaboration), J. High Energy Phys. 11 (2018) 115.

[44] A. Belyaev, N. D. Christensen, and A. Pukhov, Comput. Phys. Commun. 184, 1729 (2013).

[45] J. Pumplin, D. R. Stump, J. Huston, H. L. Lai, P. M. Nadolsky, and W. K. Tung, J. High Energy Phys. 07 (2002) 012.

[46] T. Sjostrand, S. Mrenna, and P. Z. Skands, J. High Energy Phys. 05 (2006) 026.

[47] J. Conway, R. Culbertson, R. Demina, B. Kilminster, M. Kruse, S. Mrenna, J. Nielsen, M. Roco, A. Pierce, J. Thaler, and T. Wizansky, http://conway.physics.ucdavis .edu/research/software/pgs/pgs4-general.htm.

[48] E. Conte, B. Fuks, and G. Serret, Comput. Phys. Commun. 184, 222 (2013).

[49] A. Arhrib, R. Benbrik, H. Harouiz, S. Moretti, and A. Rouchad, arXiv:1810.09106. 